\title{
Method of Calculation of Stresses in the Layered Elastic-Creeping Arrays
}

\author{
Tatiana N. Bobyleva, ${ }^{1, *}$ \\ ${ }^{1}$ Moscow State University of Civil Engineering, 129337, Yaroslavskoye sh. Moscow, Russia
}

\begin{abstract}
Stress calculation problem in an elastically creeping layered array is solved by the method of homogenization. Exponential function of time is taken as the kernel of creep of each layer. Experiments prove that such functions are the best way to describe the creep of mountain ranges over long periods of time. Integrals of convolution type with such exponential kernels are used to describe the creep process. The article contains formulas for calculating effective elastic-creep coefficients. These formulas contain exponential functions with both initial parameters and with new ones, which are also negative real numbers. As a computational example, kernel creep graph is shown for one of the average environment's effective modulus. Obtained solution can be used in underground construction.
\end{abstract}

\section{Introduction}

Most of the existing in nature and man-made materials are characterized by a heterogeneous composition. Numerous experimental studies show that the properties of structurally inhomogeneous materials (e.g., rocks, composite materials) can considerably differ from the properties of the individual components included in their structure. These issues are important in solving practical problems that arise during the construction and exploitation of buildings, the operation of composite materials. The construction of structures on rock foundations and inside rocks is an actual problem, which requires knowledge of the properties of rocks and their reaction to those or other impacts. Partial differential equations that describe the behavior of such heterogeneous materials contain rapidly changing coefficients. The method of asymptotic averaging can be used to solve such problems. This method allows to find the effective mechanical properties of heterogeneous material using the values of the mechanical characteristics of its components and their geometry. Some model structure (the periodic cell) is allocated in the array. As a result, the investigated area is considered as a periodic system with known characteristics of the individual components. To solve this problem, we can consider the environment as a macro-homogeneous, which obeys the averaged equations with constant coefficients. These coefficients are called effective. In general, the obtained homogeneous medium is anisotropic, even if the original layers were isotropic. This method of averaging is developed in the works [1-9]. In this article the characteristics of the original array (elastic

\footnotetext{
*Corresponding author: tatyana2211@,outlook.com
} 
modulus, coefficients of creep kernels, specific weight) are the step functions, abruptly changing from layer to layer. Problems for layered materials with continuously changing characteristics have been solved in [10 -12].

\section{Problem specification and decision}

We use the method of homogenization to calculate the coefficients in the constitutive stress-strain relations of elastic-creeping array. The array has a plane parallel pairs of alternating layers (Fig.1).

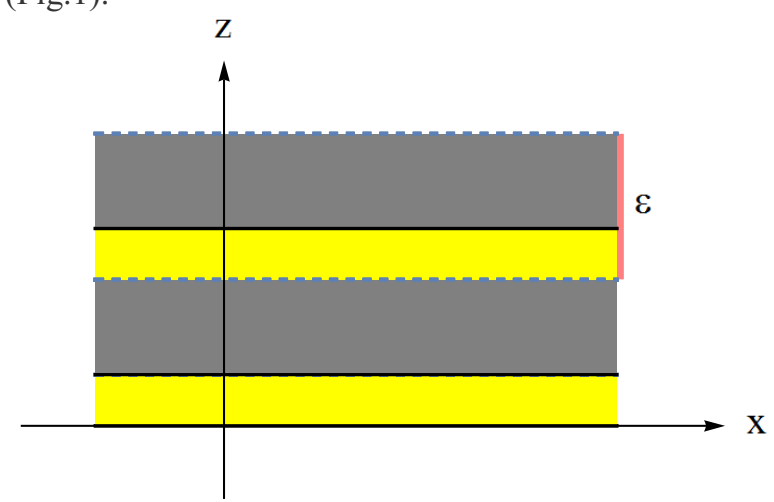

Fig. 1. Layered elastic-creeping array.

Each layer consists of isotropic elastic-creeping material.

The thickness ratio of one layer to the cell period $\varepsilon$ is denoted by $h$. Consequently, the ratio of the thickness of the other layer to the cell period will be $1-h .(0<h<1)$.

The original constitutive relations are [13]:

$$
-\frac{\partial}{\partial x_{k}}\left(a_{i j k h} \delta(t)+d_{i j k h}\right) * \frac{\partial u_{j}}{\partial x_{h}}=f_{i}(x, t),(i, j, k, h=1 \div 3) .
$$

In (1) the symbol * denotes the convolution operator:

$$
\left(d_{i j k h}(x, t) * \frac{\partial u_{j}}{\partial x_{k}}\right)=\int_{0}^{t} d_{i j k h}(x, t-\tau) \frac{\partial u_{j}(x, \tau)}{\partial x_{k}} d \tau
$$

$u_{j}(j=1,2,3)$ are components of the displacement, $\delta(t)$ is Dirac-delta function, operation $\left(a_{i j k h} \delta(t)\right) *$ is equivalent to multiplication by $a_{i j k h}$. (Einstein convention for repeated indices is used.) We are considering isotropic materials therefore the tensors in (1) can be expressed in the form $[1-3,13,14]$ :

$$
\begin{gathered}
a_{i j k h}=\lambda \delta_{i j} \delta_{k h}+\mu\left(\delta_{i k} \delta_{j h}+\delta_{i h} \delta_{j k}\right) \\
d_{i j k h}=-\left(G_{v}(t)-\frac{1}{3} G_{s}(t)\right) \delta_{i j} \delta_{k h}-\frac{1}{2} G_{s}(t)\left(\delta_{i k} \delta_{j h}+\delta_{i h} \delta_{j k}\right) .
\end{gathered}
$$


We denote here by $G_{s}$ and $G_{v}$ the regular part of the shear and the bulk relaxation respectively, by $\delta_{\mathrm{ij}}$ Kronecker symbol. Let us assume that the amplitude of the bulk creeping kernel is proportional to the shear amplitude with a coefficient of proportionality $k_{i}$ for each layer that is: $\left(G_{v}\right)_{i}=k_{i}\left(G_{s}\right)_{i}, \quad k_{i}=\mathrm{const}, k>0, \quad(i=1,2)$. Further, $G_{s}$ is denoted by $G$.

As usual we denote by $\lambda_{i}, \mu_{i}$ Lame parameters, by $\gamma_{i}$ specific weight, by $G_{i}$ creeping kernel for each layer $(i=1,2)$.

In this problem, all elastic modulus, specific weight and creeping kernels are periodic functions of the coordinate $\xi=\frac{z}{\varepsilon}$ ( $\varepsilon$ is the cell period) and are piecewise constant functions of this variable, i.e., elastic modulus, specific weight and creep kernels have the form:

$$
\begin{gathered}
\lambda_{i}=\left\{\begin{array}{l}
\lambda_{1}, \xi \in[0 ; h], \\
\lambda_{2}, \xi \in[1-h ; 1],
\end{array} \quad \mu_{i}=\left\{\begin{array}{l}
\mu_{1}, \xi \in[0 ; h], \\
\mu_{2}, \xi \in[1-h ; 1],
\end{array} \gamma_{i}=\left\{\begin{array}{l}
\gamma_{1}, \xi \in[0 ; h], \\
\gamma_{2}, \xi \in[1-h ; 1],
\end{array}\right.\right.\right. \\
G_{i}=\left\{\begin{array}{l}
G_{1}, \xi \in[0 ; h], \\
G_{2}, \xi \in[1-h ; 1],
\end{array} \quad 0<h<1, i=1,2 .\right.
\end{gathered}
$$

Let us choose creep kernel of exponential type for each layer: $G_{i}=g_{i} e^{-\alpha_{i} t}$, where $\alpha_{i}, g_{i}$ are the constants, $\alpha_{i}>0, g_{i}>0,(i=1,2), t$ is the variable that specifies the time.

We apply the Laplace transform in the time domain to the equations (1)

$$
\tilde{f}(p)=\int_{0}^{\infty} f(t) e^{-p t} d t
$$

The result is the system of elasticity theory with a complex parameter $\mathrm{p}$. We apply to this system the homogenization method described in $[1,2]$. After that we use the inverse Laplace transform and obtain the following symmetric matrix of effective coefficients for the elastic creeping array:

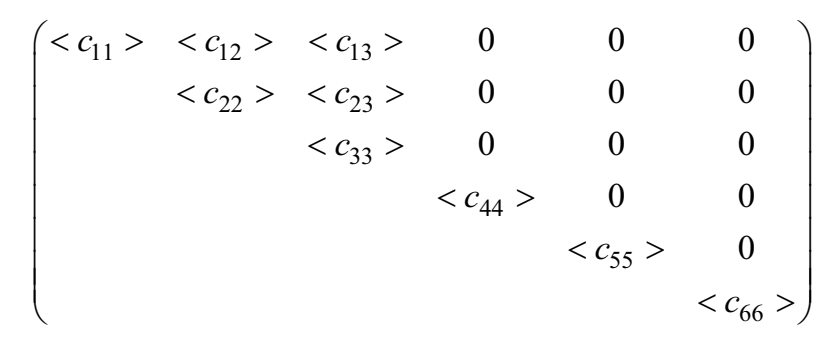

In the next formulas we have denoted for brevity:

$$
L_{i}=\lambda_{i}+2 \mu_{i}, G_{i}=g_{i}\left(k_{i}+\frac{2}{3}\right), j_{i}=g_{i}\left(k_{i}-\frac{1}{3}\right), i=1,2
$$

We have got a transversely isotropic homogeneous medium with the next effective modulus: 


$$
\begin{aligned}
& <c_{11}>=<c_{22}>=-\frac{(1-h) h}{L}\left\{\frac{e^{p_{1} t}\left[\left(\alpha_{1}+p_{1}\right)\left(j_{2}-\lambda_{2}\left(\alpha_{2}+p_{1}\right)\right)-\left(\alpha_{2}+p_{1}\right)\left(j_{1}-\lambda_{1}\left(\alpha_{1}+p_{1}\right)\right)\right]^{2}}{\left(\alpha_{1}+p_{1}\right)\left(\alpha_{2}+p_{1}\right)\left(p_{1}-p_{2}\right)}+\right. \\
& +\frac{e^{p_{2} t}\left[\left(\alpha_{1}+p_{2}\right)\left(j_{2}-\lambda_{2}\left(\alpha_{2}+p_{2}\right)\right)-\left(\alpha_{2}+p_{2}\right)\left(j_{1}-\lambda_{1}\left(\alpha_{1}+p_{2}\right)\right)\right]^{2}}{\left(\alpha_{1}+p_{2}\right)\left(\alpha_{2}+p_{2}\right)\left(p_{2}-p_{1}\right)}+\frac{e^{-\alpha_{1} t} j_{1}^{2}\left(\alpha_{2}-\alpha_{1}\right)}{\left(\alpha_{1}+p_{1}\right)\left(\alpha_{1}+p_{2}\right)}+ \\
& \left.+\frac{e^{-\alpha_{2} t} j_{2}^{2}\left(\alpha_{1}-\alpha_{2}\right)}{\left(\alpha_{2}+p_{1}\right)\left(\alpha_{2}+p_{2}\right)}+\left(\lambda_{1}-\lambda_{2}\right)^{2} \delta(t)\right\}+\left(L_{1}+L_{2}\right) \delta(t)-e^{-\alpha_{1} t} G_{1} h-e^{-\alpha_{2} t} G_{2}(1-h) .
\end{aligned}
$$

Here $p_{1}, p_{2}$ are the roots of the next quadratic equation:

$$
L p^{2}+\left[L\left(\alpha_{1}+\alpha_{2}\right)-\left((1-h) G_{1}+h G_{2}\right)\right] p+\left[L \alpha_{1} \alpha_{2}-G_{2} h \alpha_{1}-G_{1}(1-h) \alpha_{2}\right]=0 .
$$

This equation has a positive discriminant:

$$
D=\left[G_{2} h-G_{1}(1-h)+L\left(\alpha_{1}-\alpha_{2}\right)\right]^{2}+4 G_{1} G_{2} h(1-h),
$$

consequently the roots $p_{1}, p_{2}$ will always be real. (We denote in (10): $L=(1-h) L_{1}+h L_{2}$ ).

$$
\begin{aligned}
& <c_{12}>=-\frac{(1-h) h}{(1-h) L_{1}+h L_{2}}\left\{\frac{e^{p_{1} t}\left[\left(\alpha_{1}+p_{1}\right)\left(j_{2}-\lambda_{2}\left(\alpha_{2}+p_{1}\right)\right)-\left(\alpha_{2}+p_{1}\right)\left(j_{1}-\lambda_{1}\left(\alpha_{1}+p_{1}\right)\right)\right]^{2}}{\left(\alpha_{1}+p_{1}\right)\left(\alpha_{2}+p_{1}\right)\left(p_{1}-p_{2}\right)}+\right. \\
& +\frac{e^{p_{2} t}\left[\left(\alpha_{1}+p_{2}\right)\left(j_{2}-\lambda_{2}\left(\alpha_{2}+p_{2}\right)\right)-\left(\alpha_{2}+p_{2}\right)\left(j_{1}-\lambda_{1}\left(\alpha_{1}+p_{2}\right)\right)\right]^{2}}{\left(\alpha_{1}+p_{2}\right)\left(\alpha_{2}+p_{2}\right)\left(p_{1}-p_{2}\right)}+\frac{e^{-\alpha_{1} t} j_{2}^{2}\left(\alpha_{2}-\alpha_{1}\right)}{\left(\alpha_{1}+p_{1}\right)\left(\alpha_{1}+p_{2}\right)}+ \\
& \left.+\frac{e^{-\alpha_{2} t} j_{2}^{2}\left(\alpha_{1}-\alpha_{2}\right)}{\left(\alpha_{2}+p_{1}\right)\left(\alpha_{2}+p_{2}\right)}+\left(\lambda_{1}-\lambda_{2}\right)^{2} \delta(t)\right\}+\left(\lambda_{1}+\lambda_{2}\right) \delta(t)-e^{-\alpha_{1} t} j_{1} h-e^{-\alpha_{2} t} j_{2}(1-h) \\
& <c_{33}>=\frac{1}{L}\left\{\frac{e^{p_{1} t}\left[G_{1}-L_{1}\left(\alpha_{1}+p_{1}\right)\right]\left[G_{2}-L_{2}\left(\alpha_{2}+p_{1}\right)\right]}{p_{1}-p_{2}}-\right. \\
& \left.-\frac{e^{p_{2} t}\left[G_{1}-L_{1}\left(\alpha_{1}+p_{2}\right)\right]\left[G_{2}-L_{2}\left(\alpha_{2}+p_{2}\right)\right]}{p_{1}-p_{2}}+L_{1} L_{2} \delta(t)\right\} \\
& <c_{13}>=<c_{23}>=\frac{1}{L^{2}\left(p_{1}-p_{2}\right)}\left\{L\left[(1-h) j_{2} L_{1}+h L_{2}\left(j_{1}-\lambda_{1}\left(\alpha_{1}-\alpha_{2}\right)\right)\right]\left[e^{p_{1} t}\left(\alpha_{1}+p_{1}\right)-e^{p_{2} t}\left(\alpha_{1}+p_{2}\right)\right]-\right. \\
& \left.-L\left[G_{2} h j_{1}+G_{1}(1-h) j_{2}\right]+h(1-h)\left(\lambda_{1}-\lambda_{2}\right)\left[G_{2} L_{1}\left(\alpha_{1}+p_{1}\right)-G_{1} L_{2}\left(\alpha_{2}+p_{1}\right)\right]\left(e^{p_{1} t}-e^{p_{2} t}\right)\right\}+
\end{aligned}
$$




$$
+\frac{L_{1} \lambda_{2}(1-h)+h \lambda_{1} L_{2}}{L} \delta(t)
$$

In (11-13) $p_{1}, p_{2}$ are the roots of the equation (9).

$$
\begin{aligned}
<c_{44}>=<c_{55}> & =\frac{1}{\left[(1-h) \mu_{1}+h \mu_{2}\right]\left(p_{1}-p_{2}\right)}\left\{e ^ { p _ { 1 } t } [ g _ { 1 } - \mu _ { 1 } ( \alpha _ { 1 } + p _ { 1 } ) ] \left[g_{2}-\mu_{2}\left(\alpha_{2}+p_{1}\right)-\right.\right. \\
& \left.-e^{p_{2} t}\left[g_{1}-\mu_{1}\left(\alpha_{1}+p_{2}\right)\right]\left[g_{2}-\mu_{2}\left(\alpha_{2}+p_{2}\right)\right]\right\}+\frac{\mu_{1} \mu_{2} \delta(t)}{(1-h) \mu_{1}+h \mu_{2}}
\end{aligned}
$$

Here $p_{1}, p_{2}$ are the roots of the next quadratic equation:

$$
M p^{2}+\left[M\left(\alpha_{1}+\alpha_{2}\right)-\left((1-h) g_{1}+h g_{2}\right)\right] p+\left[M \alpha_{1} \alpha_{2}-g_{2} h \alpha_{1}-g_{1}(1-h) \alpha_{2}\right]=0
$$

which also has a positive discriminant. (We denote in (14): $M=2\left[(1-h) \mu_{1}+h \mu_{2}\right]$ ).

$$
<c_{66}>=\frac{1}{2}\left(c_{11}-c_{12}\right)=-e^{-\alpha_{1} t} g_{1} h-e^{-\alpha_{2} t} g_{2}(1-h)+\left[h \mu_{1}+(1-h) \mu_{2}\right] \delta(t)
$$

The calculation takes the following values of Lamé constants and the parameters of the creep kernels for each layer $[15,16]$ :

$$
\begin{aligned}
& \lambda_{1}=288,46 \mathrm{MPa}, \lambda_{2}=2019 \mathrm{MPa}, \mu_{1}=192,3 \mathrm{MPa}, \mu_{2}=1346 \mathrm{MPa}, k_{1}=k_{2}=0,7, \\
& g_{1}=17,3 \times 10^{-4} \text { (hour) }^{-1}, g_{2}=3,45 \times 10^{-4} \text { (hour) }^{-1}, \alpha_{1}=0,003, \alpha_{2}=0,0015 .
\end{aligned}
$$

As a result, for example, we plotted creep kernel $g_{11}$ containing exponential functions depending on time $t$ (hours) for $t \in[0 ; 5000]$ (Fig. 2). This creep kernel is included in the average modulus $\left\langle c_{11}\right\rangle$.

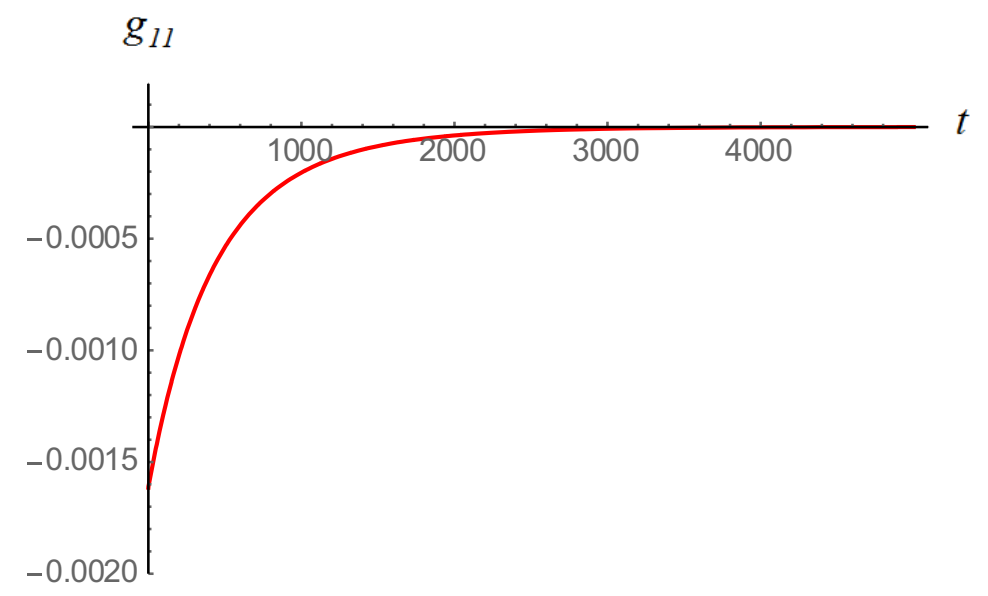

Fig. 2. Creep kernel $g_{11}$ after homogenization. 


\section{Conclusion}

Received formulas give the explicit analytical solution of the problem of calculating displacements in elastic creeping layered array. These explicit expressions can be useful in structural mechanics for example in underground construction and in problems of the science of materials, in particular, for the deformation analysis of layered polymers over large intervals of time.

Considered convolution kernels are sums of exponential functions. Such kernels are sometimes called spectral. In this paper the spectral kernels containing only one's own exponential function describe each of the two original materials. By the methods of this work it is easy to prove that if the kernel of creep of each material was set with sums of a finite number of exponential functions, then effective kernels of creep for the averaged material were also represented by the sums of a finite number of exponential functions. In this case indicators of exponential functions are roots of some polynomial, and the coefficients of this polynomial can be explicitly calculated by using the characteristics of the original two materials and the geometrical parameters of the layers.

\section{References}

1. O.A. Oleynik, A.S. Shamaev, G.A. Yosifian, Mathematical Problems in Elasticity and Homogenization (1992)

2. D.I. Bardzokas, A.I. Zobnin, Mathematical Modelling of Physical Processes in Composite Materials of Periodical Structures (2003)

3. B.E. Pobedrya, Mechanics of Composite Materials (1984)

4. A.S. Shamaev, V.V. Shumilova, Doklady Physics 56 (1) 43 (2011) doi: 10.1134/S1028335811010083

5. A.S. Shamaev, V.V. Shumilova, Fluid Dynamics $46 \quad$ (2), 250 (2011) doi: $10.1134 / \mathrm{S} 0015462811020074$

6. E. Sanchez-Palencia, Non-Homogeneous Media and Vibration Theory (1980)

7. A.S. Shamaev, V.V. Shumilova, Proceedings of the Steklov Institute of Mathematics 295, 23 (2016)

8. A.S. Shamaev, V.V. Shumilova, Proceedings of the Steklov Institute of Mathematics 295, 35 (2016)

9. T. Bobyleva, Procedia Engineering 153, 103 (2016) doi: 10.1016/j.proeng.2016.08.087

10. V.I. Andreev, D.A. Kapliy, Procedia Engineering 111, 36 (2015) doi: 10.1016/j.proeng.2015.07.032

11. V.I. Andreev, Some problems and methods of mechanics inhomogeneous bodies (2002)

12. N.Yu.Tsybina, R.A.Turusova, V.I. Andreev, Procedia Engineering 153, 51 (2016) doi: 10.1016/j.proeng.2016.08.079

13. A.A. Ilyushin, B.E. Pobedrya, Foundations of the mathematical theory of thermoviscoelasticity (1970)

14. Yu.N. Rabotnov, Creep of Structural Elements (1966)

15. S.A. Konstantinova, V.M. Pestrenin, I.V. Pestrenina, Proceedings of higher educational institution, Mining journal 4, 92 (2007)

16. M. H. Gabdrahimov, M.N. Dedyukin, Research on mining. The proceedings of the conference on the results of scientific research (1968) 